\title{
Erectile dysfunction in patients taking psychotropic drugs and treated with phosphodiesterase-5 inhibitors
}

\author{
Rossella Mazzilli ${ }^{1}$, Gloria Angeletti ${ }^{2}$, Soraya Olana ${ }^{1}$, Michele Delfino ${ }^{1}$, Virginia Zamponi ${ }^{1}$, \\ Chiara Rapinesi $^{2}$, Antonio Del Casale ${ }^{2}$, Georgios D. Kotzalidis ${ }^{2}$, Jlenia Elia ${ }^{1}$, Gemma Callovini ${ }^{2}$, \\ Paolo Girardi ${ }^{2}$, Fernando Mazzilli ${ }^{1}$ \\ ${ }^{1}$ Andrology Unit, Sant'Andrea Hospital, Sapienza University of Rome, School of Medicine and Psychology, Rome, Italy; \\ ${ }^{2}$ NESMOS (Neurosciences, Mental Health, and Sensory Organs) Department, Sant'Andrea Hospital, Sapienza University of Rome, \\ School of Medicine and Psychology, Rome, Italy.
}

\begin{abstract}
Summary Objectives: The aim of this study was to assess the prevalence of patients with Erectile Dysfunction (ED) receiving psychotropic drugs, the impact of these drugs on hormonal profile, and the efficacy of PDE5-i in these patients.

Materials and methods: We recruited 1872 patients referring for ED to our Andrology Unit. Assessment included serum testosterone, gonadotropins, TSH, prolactin, and PSA, and the IIEF-5 questionnaire for ED diagnosis. Inclusion criteria were age 21-75 years and IIEF-5 total score $\leq 21$; exclusion criteria included hypogonadism, diabetes mellitus, previous prostatectomy, other medication intake, and ED diagnosis prior to psychotropic drug treatment. Efficacy was rated with the IIEF-5 (remission: total score $\geq 22$ ).

Results: The prevalence of ED patients treated with psychotropic drugs since $\geq 3$ months was 9.5\% (178/1872), subdivided according to the drugs used into: Group A, 16 patients treated with atypical antipsychotics (9.0\%); Group B, 55 patients with benzodiazepines (30.9\%); Group C, 33 patients with antidepressant drugs (18.5\%); and Group D, 74 patients with multiple psychotropic drugs (41.6\%). Patients in Group A were significantly younger than other groups $(p<0.05)$. The hormonal profile presented only higher prolactin level in patients treated with antipsychotics, alone or in combination $(p<0.05)$.

Overall, 146 patients received PDE5-i. Remission rate, after three months of treatment, was significantly higher in Group B compared to $C$ and $D$ groups $(p<0.05)$.

Conclusions: A substantial portion of patients receiving psychotropic drugs show ED. Sexual performance in these patients benefits from PDE5-i. Age, effects of psychiatric disorders, psychotropic drugs, and PDE5-i treatment modality accounted for variability of response in this sample.
\end{abstract}

KEY WORDS: Erectile dysfunction; Psychotropic drugs; Phosphodiesterase 5-inhibitors; Antipsychotic drugs; Antidepressant drugs; Benzodiazepines.

Submitted:14 December 2017; Accepted 20 February 2018

\section{INTRODUCTION}

Erectile Dysfunction (ED) is defined as the persistent inability to achieve or maintain penile erection that is sufficient for satisfactory sexual performance. Organic (neurological, endocrine-metabolic disorders, diabetes, vascular, and mechanic), psychological and pharmacological (e.g., drug interference) factors may lead to ED (1). The drug classes most frequently associated with ED are antihypertensive, alpha-blockers, proton pump inhibitors and psychotropic drugs, especially antidepressants and antipsychotics.

There are many ways by which drugs can interfere with the neuro-vascular and sensory mechanisms of sexual function (2). In particular, antipsychotics and serotonin reuptake inhibitors (SSRIs), in addition to anorgasmia and decreased libido, may induce ED, mainly through dopamine inhibition and the consequent increase in prolactin levels. However, antipsychotics differ in their hyperprolactinemic effect; risperidone, olanzapine, ziprasidone, and amisulpride have all been associated with hyperprolactinemia, quetiapine, clozapine and aripiprazole with both hyperprolactinemia and its reversal, and lurasidone has shown no hyperprolactinemizing potential.

Furthermore, combining antidepressants with antipsychotics was associated with increased prolactin level (3).

There are several reports and studies dealing with the treatment of ED with PDE5-i in patients receiving psychotropic medication, mainly in patients who developed ED secondary to antidepressant or antipsychotic intake. Antidepressants are long known to be associated with sexual side effects (2) and more than half of the patients who receive antidepressants (Ads) develop sexual side effects. The main mechanisms by which antidepressants affect sexual function are monoaminergic, i.e., serotonergic, noradrenergic, and dopaminergic.

Almost all currently used antidepressants acutely increase the concentration of biogenic amines in the synaptic cleft and in the longer term down-regulate serotonergic and noradrenergic receptors in the synapses. In man, cavernosal serotonin mediates contraction, hence reduced penile blood influx, with 5-HT1A, 5-HT2A, and 5-HT4 serotonin receptor stimulation inducing detumescence that may be blocked by antagonists of these receptors.

To further add to this complexity, dopamine D1 and D2like receptor agonists strain-dependently induce penile erection (4). Antipsychotic drugs, both classical and atypical, are able to block dopaminergic function by inhibiting all types of dopamine receptors, so their ability to cause ED is not surprising (5). Increased intracellular cGMP is crucial for erection; various phosphodiesterases (PDEs) hydrolyze cGMP (PDE5, 6 and 9 specifically) to 5'-GMP thereby decreasing its intracellular concentration and consequently, erection, thus providing an adequate rationale for PDE inhibitors in the treatment of ED. The effects of 
sildenafil have been evaluated and found satisfactory in patients treated with antipsychotics who had developed $\mathrm{ED}$, including atypical antipsychotics, such as olanzapine and risperidone or antidepressant drugs; in all studies, sildenafil was effective and well-tolerated (6-9). Tadalafil was also found to be effective in patients treated with antidepressants, like serotonin reuptake inhibitors (SSRIs) (10). However, no studies to date focused conjointly on how many patients with ED were treated with psychotropics and on their endocrine derangement.

We aimed to retrospectively investigate: a) the prevalence of patients who are treated with psychotropic drugs among men with ED; b) the impact of these drugs on their hormonal profile; and c) the efficacy of PDE5-i in relieving ED in patients with psychotropic-associated ED who continue on their psychotropic medication.

\section{Materials AND METHOdS}

\section{Patients}

We recruited 1872 patients with ED between 21 and 75 years of age who referred to our Andrology Unit (Sant'Andrea Hospital-Sapienza University, Rome, Italy) between January 2006 and July 2017. Patients were explained the purpose of the study and signed free, informed consent. Assessment comprised medical history collection, physical examination, in particular andrological, and the completion of a sociodemographic data collection sheet. All patients completed the International Index of Erectile Function (IIEF-5) questionnaire (11). Those scoring $\leq 21$ were eligible for the study. Exclusion criteria comprised previous radical prostatectomy, hypogonadism in treatment with testosterone, diagnosis of diabetes mellitus (DM), based on fasting plasma glucose and glycosylated hemoglobin-HbAlc levels, a previous PDE5-i treatment, a concomitant treatment with other, non -psychotropic medications. At baseline we obtained blood to evaluate luteinizing hormone (LH), follicle stimulating hormone (FSH), testosterone, thyroid stimulating hormone (TSH), prolactin, and prostate specific antigen (PSA).

Hormonal assays. Between h 08:00 and 11:00 we withdrew from each patient $5 \mathrm{~mL}$ of blood in $5 \mathrm{~mL}$ vials. The vials were centrifuged at 3,000 r.p.m. for $10 \mathrm{~min}$ and kept at $20^{\circ} \mathrm{C}$ until the day of the assay. Assays were carried-out using enzyme-linked fluorescence assay (ELFA); we investigated serum testosterone, FT4, TSH, and prolactin.

Psychometric assessment. The patients completed the validated Italian version of the abridged IIEF-5 questionnaire, consisting of five items each rated on a 5-point Likert scale ranging from 1 (low on sexuality) to 5 (normal sexual performance) (11). A score of $\leq 21$ is considered as showing the existence of ED, while scores of $\geq 22$ are considered as remission.

Treatment. In the absence of contraindications, included ED patients had the option to choose between an "on demand" schedule of a PDE5-i, i.e., 50-100 mg oral sildenafil, 10-20 mg vardenafil, $10-20 \mathrm{mg}$ tadalafil, and (since 2013) $100-200 \mathrm{mg}$ avanafil, or a $5 \mathrm{mg} /$ day oral tadalafil "once a day" schedule. In particular, the "on demand" drug was chosen with the patient, according to time of onset, duration of action, and interactions with food and alcohol, which are specific for each PDE5-i. The study adhered to the Hospital's Ethics Committee guidelines and to the Ethical Principles for Medical Research Involving Human Subjects as adopted at the $18^{\text {th }}$ WMA General Assembly, Helsinki, Finland, June 1964, and amended by the $55^{\text {th }}$ WMA General Assembly, Tokyo, Japan, October 2004 and subsequent modifications when enforced (last, Fortaleza, Brazil, October 2013).

\section{Statistics}

Given the large sample size, we used parametric statistics to analyze our data. We used the paired t-test and Fisher's exact test for continuous and categorical data, respectively. Continuous data were described as absolute values, mean \pm Standard Deviation (SD), and range. Categorical data were described as absolute, percentage frequency, and 95\% Confidence Intervals (CIs). We set statistical significance at $p<0.05$. Analyses were carriedout with the Statistical Package for the Social Sciences (SPSS), version 24 (International Business Machines Corporation [IBM], Armonk, New York, US).

\section{RESULTS}

Of the 1872 evaluated patients, 1642 were not receiving any psychotropic medication, while 230 patients (12.3\%) were receiving psychotropic drugs; of them, 52 were excluded, 9 because they were previously prostatectomized, 8 were hypogonadic in treatment with testosterone, 14 had DM, and 47 were taking also non-psychotropic medications, thus leaving a final sample of 178 (9.5\%). The sample's sociodemographic characteristics are shown in Table 1.

The 178 patients were distributed into 4 groups, according to the psychotropic drugs they used (Table 2):

- Group A: 16/178 patients (9.0\%) were treated with atypical antipsychotics (olanzapine, aripiprazole, risperidone and quetiapine);

\section{Table 1.}

Sociodemographic characteristics of the included men $(N=178) . S D$, standard deviation.

\begin{tabular}{|lcc|}
\hline & Mean (range) & SD \\
\hline Age & $51.6(21-75)$ & 13.1 \\
\hline Marital status & $\mathbf{N}$ & $\mathbf{( \% )}$ \\
Single & 43 & \\
Married & 97 & 54.2 \\
Divorced & 25 & 14.0 \\
Widowed & 13 & 7.3 \\
\hline Educational level & & \\
Primary school & 3 & 1.7 \\
High School & 15 & 8.4 \\
Superior high school & 83 & 46.7 \\
College/University & 54 & 30.3 \\
Specialization/Master/Ph.D. & 23 & 12.9 \\
\hline Occupational status & & \\
Unemployed & 21 & 11.8 \\
Blue collar worker & 28 & 15.7 \\
Employee & 44 & 24.8 \\
Entrepreneur & 28 & 15.7 \\
Free-lance professional & 42 & 23.6 \\
Executive & 15 & 8.4 \\
\hline
\end{tabular}




\begin{tabular}{|c|c|c|c|c|c|c|c|c|}
\hline & $N=178$ & $\begin{array}{l}\text { Age (years) } \\
\text { (range) }\end{array}$ & $\begin{array}{c}\text { LH } \\
(\mathrm{mlU} / \mathrm{ml})\end{array}$ & $\begin{array}{c}\text { FSH } \\
(\mathrm{mlU} / \mathrm{ml})\end{array}$ & $\begin{array}{c}\text { Testosterone } \\
(\mathrm{ng} / \mathrm{ml})\end{array}$ & $\begin{array}{c}\text { TSH } \\
(\mathrm{mlU} / \mathrm{ml})\end{array}$ & $\begin{array}{l}\text { Prolactin } \\
\text { (ng/ml) }\end{array}$ & $\begin{array}{c}\text { PSA } \\
(\mathrm{ng} / \mathrm{ml})\end{array}$ \\
\hline Group A & 16 & $\begin{array}{c}42.5 \pm 14.1^{*} \\
(25-59)\end{array}$ & $\begin{array}{l}5.2 \pm 2.8 \\
(2.7-9.2)\end{array}$ & $\begin{array}{l}5.1 \pm 2.6 \\
(3.1-8.0)\end{array}$ & $\begin{array}{c}5.3 \pm 2.0 \\
(3.1-8.0)\end{array}$ & $\begin{array}{l}1.6 \pm 0.5 \\
(0.92-2.3)\end{array}$ & $\begin{array}{c}16.9 \pm 2.8^{*} \\
(14.0-21.3)\end{array}$ & $\begin{array}{c}1.4 \pm 1.1 \\
(0.58-2.15)\end{array}$ \\
\hline Group B & 55 & $\begin{array}{c}54.0 \pm 13.5 \\
(24-75)\end{array}$ & $\begin{array}{c}4.9 \pm 2.3 \\
(2.3-8.7) \\
\end{array}$ & $\begin{array}{l}4.4 \pm 1.5 \\
(1.8-7.4) \\
\end{array}$ & $\begin{array}{l}5.9 \pm 2.3 \\
(2.7-8.8) \\
\end{array}$ & $\begin{array}{l}1.6 \pm 0.8 \\
(1.1-3.4) \\
\end{array}$ & $\begin{array}{l}6.8 \pm 3.2 \\
(4.0-11.2) \\
\end{array}$ & $\begin{array}{c}1.7 \pm 1.2 \\
(0.3-3.3) \\
\end{array}$ \\
\hline Group C & 33 & $\begin{array}{c}55.3 \pm 11.7 \\
(0.6-2.9) \\
\end{array}$ & $\begin{array}{c}5.8 \pm 2.7 \\
(36-75)\end{array}$ & $\begin{array}{l}6.6 \pm 2.6 \\
(2.0-11.2)\end{array}$ & $\begin{array}{l}5.9 \pm 1.5 \\
(3.4-12.6)\end{array}$ & $\begin{array}{c}1.0 \pm 0.8 \\
(4.6-7.9)\end{array}$ & $\begin{array}{l}7.2 \pm 3.7 \\
(0.3-1.9)\end{array}$ & $\begin{array}{l}1.6 \pm 1.1 \\
(4.9-13.3)\end{array}$ \\
\hline Group D & 74 & $\begin{array}{c}50.2 \pm 12.4 \\
(21-75)\end{array}$ & $\begin{array}{l}6.0 \pm 4.3 \\
(1.4-12.6)\end{array}$ & $\begin{array}{l}7.3 \pm 3.9 \\
(2.5-12.1)\end{array}$ & $\begin{array}{c}4.1 \pm 2.3 \\
(1.6-8.9)\end{array}$ & $\begin{array}{l}2.0 \pm 0.9 \\
(0.9-3.1)\end{array}$ & $\begin{array}{c}15.8 \pm 5.5^{*} \\
(6.8-25.4)\end{array}$ & $\begin{array}{l}1.8 \pm 1.0 \\
(0.7-1.9)\end{array}$ \\
\hline
\end{tabular}

\begin{tabular}{|c|c|c|c|}
\hline & N & Treated patients & Responders (\%) \\
\hline$\overline{\text { Total }}$ & 178 & $146 / 178(82.0 \% ; 75.7$ to 87.0$)$ & $67 / 146(45.9 \% ; 38.0$ to 54.0$)$ \\
\hline Group A & 16 & 9/16 (56.3\%; 33.2 to 76.9$)$ & $5 / 9$ (55.6\%; 26.6 to 81.2$)$ \\
\hline Group B & 55 & $47 / 55$ (85.5\%; 73.6 to 92.7$)^{*}$ & $31 / 47(66.0 \% ; 51.6 \text { to } 77.9)^{* *}$ \\
\hline Group C & 33 & $27 / 33(81.8 \% ; 65.2$ to 91.8$)$ & $11 / 27$ (40.7\%; 24.5 to 59.3$)$ \\
\hline Group D & 74 & $62 / 74(83.8 \% ; 73.6$ to 90.6$)$ & $20 / 62$ (32.3\%; 21.9 to 44.7$)$ \\
\hline
\end{tabular}

\begin{tabular}{|c|c|c|c|c|}
\hline & \multicolumn{2}{|r|}{ On demand } & \multicolumn{2}{|r|}{ Once a day } \\
\hline & $N$ & Responders (\%) & $N$ & Responders (\%) \\
\hline Total & 113 & $54 / 113$ (47.8\%; 38.8-56.9) & 33 & $14 / 33(41.2 \% ; 26.3-57.8)$ \\
\hline Group A & $7 / 10$ & $3 / 7(42.9 \% ; 15.8$ to 75.0$)$ & $3 / 10$ & $2 / 3(66.7 \% ; 20.2$ to 94.4$)$ \\
\hline Group B & $42 / 47$ & $29 / 42(69.1 \% ; 53.9 \text { to } 81.0)^{*}$ & $5 / 47$ & $3 / 5(60.0 \% ; 22.9$ to 88.4$)$ \\
\hline Group C & $16 / 27$ & $6 / 16$ (37.5\%; 18.4 to 61.5$)$ & $11 / 27$ & $4 / 11(36.4 \% ; 15.0$ to 64.8$)$ \\
\hline Group D & $48 / 62$ & $16 / 48(33.3 \% ; 21.6$ to 47.5$)$ & $14 / 62$ & $5 / 14(35.7 \% ; 16.2$ to 61.4$)$ \\
\hline
\end{tabular}

Table 2.

Age and hormonal profile in the different groups considered [mean values $\pm \mathrm{SD}$ (min.-max)].

Table 3.

Therapeutic efficacy of PDE5-i in the various psychotropic treatment groups (IIEF-5: total score $\geq 22)[\mathrm{N}(\%, 95 \% \mathrm{Cl})]$.

\section{Table 4.}

PDE5-i "on demand" or "once a day" treatment and efficacy (IIEF-5: total score $\geq 22$ ) per psychotropic treatment [N $(\%, 95 \% C l)]$.
- Group B: 55/178 patients (30.9\%) treated with benzodiazepines (alprazolam, lorazepam, clonazepam, bromazepam);

- Group C: 33/178 patients (18.5\%) treated with antidepressant drugs in monotherapy (SSRIs, i.e., fluoxetine, sertraline, citalopram, escitalopram, or paroxetine; serotonin-norepinephrine reuptake inhibitors (SNRIs), i.e., duloxetine and venlafaxine; and tricyclic antidepressants (TCAs), i.e., amitriptyline and clomipramine);

- Group D: 74/178 patients (41.6\%) in polytherapy with antidepressant drugs (citalopram, paroxetine, and clomipramine) and/or atypical antipsychotics (olanzapine, aripiprazole, and risperidone) and/or benzodiazepines (alprazolam, lorazepam, and clonazepam) and/or mood stabilizers (lithium carbonate, carbamazepine, and valproic acid).

No patient was on monotherapy with monoamine oxidase inhibitors (MAOIs) or mood stabilizers.

Age. Patients in Group A were significantly younger compared to the other groups $(\mathrm{p}<0.05)$ (Table 2$)$.

Hormonal profile. The four groups did not differ in hormone levels, except for prolactin, that was higher in patients treated with antipsychotics alone or those in polytherapy with respect to the groups treated with benzodiazepines or antidepressants alone $(\mathrm{p}<0.05)$ (Table 2).
ED Treatment. Of the 178 patients, 146 (82.0\%) accepted PDE5-i treatment; patients in group A adhered to treatment significantly more than patients in group B (p $<0.05)$. The remaining $32(18.0 \%)$ patients refused PDE5-i treatment for various reasons.

Response/remission of ED, as assessed after three months of treatment through the IIEF-5 questionnaire (i.e., a total score of $\geq 22$ ), was significantly higher in group B compared to groups C and D ( $<<0.05)$ (Tables 3-4).

Regarding PDE5-i treatment modality, 113/146 (77.4\%) patients chose "on demand" while 33/146 (22.6\%) chose "once a day" administration. "On demand" and "once a day" groups showed no significant differences in positive response rates $(47.8 \%$ vs. $41.2 \%$; $p=$ n.s.).

However, considering individual groups, response/remission in patients who chose an "on demand" modality was significantly higher in Group B, compared to Groups C and D ( $\mathrm{p}<0.05)$. Regarding the "once a day" modality, no between-groups differences were shown.

Of the 146 patients who adhered to PDE5-i treatment, $67(45.9 \%)$ responded positively to treatment (final IIEF-522).

The IIEF-5 total score varied from $12.5 \pm 3.8$ (range 621 ) at baseline to $19.8 \pm 3.8$ (range $8-25$ ) at the threemonth follow-up. The difference was statistically significant $(\mathrm{p}<0.01)$. 


\section{Discussion}

In this study we focused on identifying the prevalence of psychotropic drug use in ED patients, on observing psychotropic drug impact on ED patient's hormonal profile, and on the efficacy of PDE5-i in such patients. About $12.3 \%$ of patients with ED in our sample were receiving psychotropic medication and about $9.5 \%$ met criteria for inclusion; of them, about half achieved remission in response to PDE5-i treatment added on their psychotropic $\operatorname{drug}(\mathrm{s})$. In this cross-sectional study, we found the group receiving only benzodiazepines to fare better than groups receiving other drugs or combinations, even after splitting the sample into "on demand" or "once a day" modalities. We also found higher serum prolactin only in patients receiving antipsychotics, alone or in combination, as well as age differences among the four groups. A possible pathogenic factor of ED is represented by drug interference. Among drugs, psychotropics have an important role, even if, in the presence of $\mathrm{ED}$, the line between effect of drug therapy and psychopathology is undefined. In fact, in patients with depressive symptoms, symptoms like apathy or anhedonia, and in those with schizophrenia, specifically anhedonia, could negatively shape sexual life (5-9).

We are aware of no studies investigating the prevalence of psychotropic drug use in an ED population. Here we found a $9.5 \%$ prevalence involving patients developing ED after at least 3-month psychotropic drug use. Studies investigating psychotropic drug prescription/use in the general population worldwide have produced different results depending on the period of investigation and on the country where the search was conducted (Supplementary Table). Comparing our data with the last European data on general populations (12.3\%), our proportion of patients with ED who are on psychotropics is similar. However, given that in most studies significantly more women than men use psychotropics, and considering that our sample was composed of men only, we might suppose that psychotropic drug use in ED populations may be higher than in the general population. We divided our sample into 4 groups according to the type of drugs they used, i.e., atypical antipsychotics, benzodiazepines, and antidepressant drugs in monotherapy and those who were on polytherapy.

These subsamples differed for age, in that patients receiving antipsychotics were significantly younger than those in the other groups, probably because the psychoses have an earlier onset and an earlier treatment initiation than other psychiatric disorders. In fact, schizophrenia has an onset around adolescence and early adulthood, while anxiety disorders and depression have a later onset and a later initiation of treatment (12). The hormonal profile (gonadotropins, testosterone and TSH levels) was similar in the different groups; on the other hand, prolactin levels, a hormone related to sexual dysfunction, was significantly higher in the groups treated with atypical antipsychotics, alone or in combination, in line with literature $(3,13)$.

There are few reports on the use of sildenafil and tadalafil in patients treated with atypical antipsychotic or antidepressant drugs in monotherapy, and they showed variable efficacy $(6,14-15)$. Our study included also people treat- ed with benzodiazepines and polytherapy, and, among PDE5-i, also vardenafil and avanafil. There are few, sporadic reports on the use of these two PDE5-is in psychotropic-associated ED. The only controlled study regarded vardenafil vs. placebo and found effectiveness for both ED and mild depression in patients receiving no psychotropic drugs, that could mean either intrinsic antidepressant properties for PDE5-is or that improved sexual performance acted on depression to decrease it (16).

Acceptance to be treated was higher in patients treated with benzodiazepines, compared to the other groups. In particular, people on benzodiazepines were significantly more likely to endorse PDE5-i treatment than patients on antipsychotics and responders among the former were significantly more likely to have endorsed PDE5-i treatment than those who received either antidepressants or polytherapy. While we are not able to demonstrate why should this occur, we could speculate that people with anxiety, who usually receive benzodiazepines (although antidepressant treatment is the standard for most anxiety disorders), experience less side effects (other than dependence) than those who receive other classes of psychotropics, thus they are more open to further drug treatment to improve their ED. Help-seeking was found to be the most important factor in benzodiazepine and antidepressant use in Europe (17), but factors prompting to further drug use were not examined. We did not investigate help-seeking specifically, but most of our patients were motivated for resolving their ED. Furthermore, people with psychosis tend to be suspicious about drug treatment and doctors and show poor adherence (18).

Furthermore, we found significantly more improvement in the group receiving benzodiazepines, indicating their ED was due to an underlying anxiety disorder rather than to their medication. Although benzodiazepines may differ in their ED-inducing potential, results from large cohorts show benzodiazepines to be associated with ED, even after adjusting for comorbidities and health behaviors or addressing possible confounders (19).

Benzodiazepine increase GABAA receptor function and GABAA receptor stimulation has been shown to reduce penile erection in rats through central mechanisms, so we could expect that people on benzodiazepines could show similar rates of ED to people taking other ED-associated psychotropic drugs (20). It is possible that in conditions of extreme anxiety, in which GABAergic activity is low, its normalization through benzodiazepines could alleviate impaired erection caused by anxiety itself. Better response in the benzodiazepine group was present also when subdividing the sample according to "on demand" or "once a day" modalities, with better response in the former, while in the latter no significance could be shown, probably due to reduced sample size. On the other hand, the unsatisfactory response of the other groups (antidepressants, antipsychotics and polytherapy) could be possibly due to both the psychopathological condition and to persistent drug interference with erectile function.

The limitations of this study is that it included only patients without prior ED who underwent psychotropic drug treatment. Thus, we cannot investigate the extent to which PDE5-i treatment may benefit patients with ED 
who need psychotropic treatment for their psychological condition. Furthermore, the design was cross-sectional and retrospective. Obviously, a prospective cohort study would be more accurate and specific.

\section{Conclusions}

This study underlines the significant prevalence of patients with ED treated with psychotropic drugs, and confirms, in line with the literature, the possibility of PDE5-i to improve the sexual performances of these patients. The variability of the therapeutic efficacy appears to depend on age, on the effects of psychiatric disorders, on the psychotropic drugs used, and on the choice of PDE5-i treatment modality.

\section{ACKNOWLEDGMENTS}

The Authors wish to thank Ms Mimma Ariano, Ms Ales Casciaro, Ms Teresa Prioreschi, and Ms Susanna Rospo, Librarians of the Sant'Andrea Hospital, School of Medicine and Psychology, Sapienza University, Rome, for rendering precious bibliographical material accessible.

\section{References}

1. Foresta C, Caretta N, Corona G, et al. Clinical and metabolic evaluation of subjects with erectile dysfunction: a review with a proposal flowchart. Int J Androl. 2009; 32:198-211.

2. Waldinger MD. Psychiatric disorders and sexual dysfunction. Handb Clin Neurol. 2015; 130:469-489.

3. Pacchiarotti I, Murru A, Kotzalidis GD, et al. Hyperprolactinemia and medications for bipolar disorder: systematic review of a neglected issue in clinical practice. Eur Neuropsychopharmacol. 2015; 25:10451059.

4. Melis MR, Argiolas A, Gessa GL. Apomorphine-induced penile erection and yawning: site of action in brain. Brain Res. 1987; 415:98-104.

5. Liu-Seifert H, Kinon BJ, Tennant CJ, et al. Sexual dysfunction in patients with schizophrenia treated with conventional antipsychotics or risperidone. Neuropsychiatr Dis Treat. 2009; 5:47-54.

6. Gopalakrishnan R, Jacob KS, Kuruvilla A, et al. Sildenafil in the treatment of antipsychotic-induced erectile dysfunction: a randomized, double-blind, placebo-controlled, flexible-dose, two-way crossover trial. Am J Psychiatry. 2006; 163:494-499.

7. Atmaca M, Kuloglu M, Tezcan E. Sildenafil use in patients with olanzapine-induced erectile dysfunction. Int J Impot Res. 2002; 14:547-549.

8. Aviv A, Shelef A, Weizman A. An open-label trial of sildenafil addition in risperidone-treated male schizophrenia patients with erectile dysfunction. J Clin Psychiatry. 2004; 65:97-103.

9. Nurnberg HG, Hensley PL, Gelenberg A, et al. Treatment of antidepressant-associated sexual dysfunction with sildenafil: a randomized controlled trial. JAMA. 2003; 289:56-64

10. Segraves RT, Lee J, Stevenson R, et al. Tadalafil for treatment of erectile dysfunction in men on antidepressants. J Clin Psychopharmacol. 2007; 27:62-66.

11. Rosen RC, Cappelleri JC, Smith MD, et al. Development and evaluation of an abriged, 5-item version of the International Index of Erectile Function (IIEF-5) as a diagnostic tool for erectile dysfunction. Int J Impot Res. 1999; 11:319-326.

12. Kessler RC, Amminger GP, Aguilar-Gaxiola S, et al. Age of onset of mental disorders: a review of recent literature. Curr Opin Psychiatry. 2007; 20:359-364.

13. Park YM, Lee SH, Lee BH, et al. Prolactin and macroprolactin levels in psychiatric patients receiving atypical antipsychotics: A preliminary study. Psychiatry Res. 2016; 239:184-189.

14. Fava M, Nurnberg HG, Seidman SN, et al. Efficacy and safety of sildenafil in men with serotonergic antidepressant-associated erectile dysfunction: results from a randomized, double-blind, placebo-controlled trial. J Clin Psychiatry. 2006; 67:240-246.

15. de Boer MK, Oolders JM, van den Heuvel ER, et al. Efficacy of tadalafil on erectile dysfunction in male patients using antipsychotics: a double-blind, placebo-controlled, crossover pilot study. J Clin Psychopharmacol. 2014; 34:380-382.

16. Rosen R, Shabsigh R, Berber M, et al. Vardenafil Study Site Investigators. Efficacy and tolerability of vardenafil in men with mild depression and erectile dysfunction: the depression-related improvement with vardenafil for erectile response study. Am J Psychiatry. 2006; 163:79-87.

17. Demyttenaere K, Bonnewyn A, Bruffaerts R, et al. Clinical factors influencing the prescription of antidepressants and benzodiazepines: results from the European study of the epidemiology of mental disorders (ESEMeD). J Affect Disord. 2008; 110:84-93

18. García S, Martínez-Cengotitabengoa M, López-Zurbano S, et al. Adherence to antipsychotic medication in bipolar disorder and schizophrenic patients: a systematic review. J Clin Psychopharmacol. 2016; 36:355-371.

19. Kupelian V, Hall SA, McKinlay JB. Common prescription medication use and erectile dysfunction: results from the Boston Area Community Health (BACH) survey. BJU Int. 2013; 112:1178-1187.

20. Melis MR, Argiolas A. Reduction of drug-induced yawning and penile erection and of noncontact erections in male rats by the activation of GABAA receptors in the paraventricular nucleus: involvement of nitric oxide. Eur J Neurosci. 2002; 15:852-860.

\author{
Correspondence \\ Rossella Mazzilli, MD \\ rossella.mazzilli@uniromal.it \\ Sant'Andrea Hospital, Sapienza University of Rome, \\ School of Medicine and Psychology \\ Via di Grottarossa 1035-1039, 00189 Rome, Italy \\ Soraya Olana, $M D$ \\ Michele Delfino, MD, PhD \\ Virginia Zamponi, MD \\ Jlenia Elia, MD \\ Fernando Mazzilli, MD \\ Andrology Unit, Sant'Andrea Hospital, Sapienza University of Rome, \\ School of Medicine and Psychology, Rome, Italy
}

Gloria Angeletti, MD

Chiara Rapinesi, MD

Antonio Del Casale, MD, PhD

Georgios D. Kotzalidis, MD, PhD

Gemma Callovini, MD

Paolo Girardi, MD

NESMOS (Neurosciences, Mental Health, and Sensory Organs)

Department, Sant'Andrea Hospital, Sapienza University of Rome,

School of Medicine and Psychology, Rome, Italy 